\title{
The Apparent Galactic Disc Scale Height
}

\author{
PAVEL KROUPA \\ Institute of Astronomy, Madingley Road, Cambridge CB3 0HA, England
}

\begin{abstract}
For stars fainter than $M_{V} \approx 6$ the density distribution perpendicular to the Galactic midplane can be approximated by an exponential fall-off with a scale height of about $270 \mathrm{pc}$. We show that even if in fact constant an apparent decrease of the scale height to about $150 \mathrm{pc}$ for stars with $M_{\mathrm{V}} \approx 17$ can be expected if all 'stars' measured in the photographic surveys are unresolved binaries. In principle measurements of the apparent scale-height allow the study of the mass-ratio distribution and the proportion among 'stars' of binary systems.
\end{abstract}

\section{INTRODUCTION}

Although not gravitationally self-consistent, an exponential distribution of stars with distance from the Galactic midplane proves to be a very useful approximation (see for example Figure 5 of Kuijken \& Gilmore 1989a). The scale height of the dominant stellar population in the disc is assumed constant at about $300 \mathrm{pc}$ for main sequence stars fainter than $M_{\mathrm{V}} \approx 6$ and is much smaller for brighter stars (Gilmore \& Reid 1983). The behaviour of the thickness of the Galactic disc with stellar spectral type is understood in terms of kinematic heating of the stellar population coupled with a strong dependency of stellar lifetime on $M_{\mathrm{V}}$.

Star-count data need to be corrected for Malmquist bias to infer true space densities. However, cosmic scatter changes with spectral type. Also, unresolved binary systems appear less distant when using photometric parallax. We prefer to model star count data not corrected for Malmquist bias, and for this purpose Kroupa (1992) developed a detailed model of the dispersion about the colourmagnitude relation as a function of absolute magnitude. In what follows we shall refer to the apparent scale height as the scale height inferred from uncorrected star-count data.

\section{THE MODEL}

We generate $4 \times 10^{6}$ systems fainter than $M_{\mathrm{V}} \approx 6$ within a distance of $300 \mathrm{pc}$ assuming an exponential density decay with a scale height $h_{\text {true }}=270 \mathrm{pc}$ (Kroupa 1992). A fraction $f$ of all systems are binary stars with uncorrelated component masses chosen from one mass function (see Kroupa et al. , this Colloquium), and the remaining $1-f$ systems are single stars. The absolute magnitude of each system is changed according to an age and chemical composition (Kroupa 1992) and the apparent V- and I-band magnitudes are calculated adopting as our Gaussian measurement uncertainty $0.04 \mathrm{mag}$. We use the colour-magnitude relation corrected for the effects of unresolved binary systems, $M_{\mathrm{V}}=2.74+3.42(V-I)$ (Kroupa 1992) for photometric parallax and the transformation from the $V$ - to the I-band.

We bin the photometric distance, $d_{\mathrm{p}}$, for 'stars' in the apparent distance range $30<d_{\mathrm{p}}<170 \mathrm{pc}$ into $10 \mathrm{pc}$ wide bins, and into eight $V-I$ colour bands: 


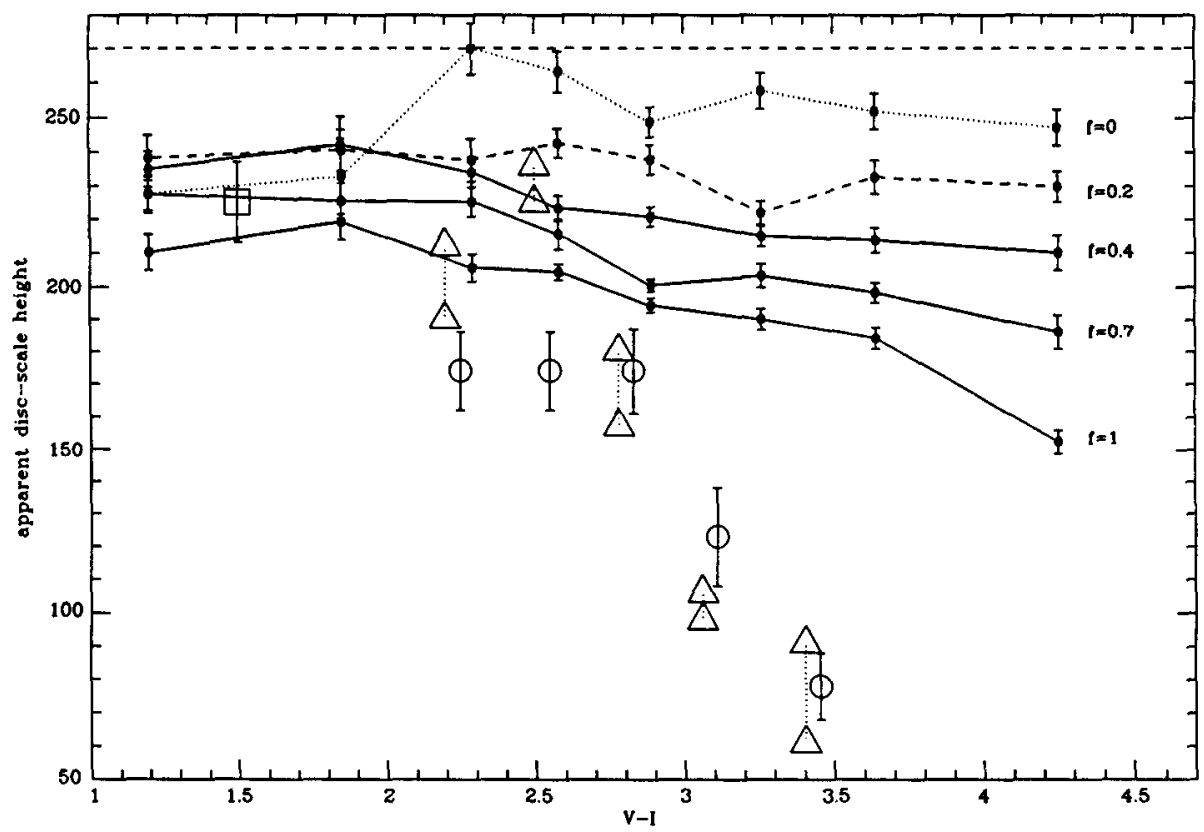

FIGURE 1. Apparent disc scale height, $h_{\mathrm{app}}$, as a function of colour for different proportions of unresolved binaries. Error bars indicate the uncertainties in each colour bin being smallest at $V-I \approx 2.7$ where the peak of the luminosity function lies. The horizontal dashed line represents our adopted disc scale height $(h=270 \mathrm{pc})$. Triangles represent the measurements of $h_{\text {mpp }}$ obtained by Hawkins (1988) for two different fields. Circles represent $h_{\text {app }}$ as a function of distance completeness limit for the SGP star-count data (Kuijken \& Gilmore 1989b) plotted next to the corresponding measurements made by Hawkins. In order of increasing $V-I$ the distance limits are $0 \leq d_{\mathrm{p}} \leq 500 \mathrm{pc}, 500 \mathrm{pc}$, $500 \mathrm{pc}, 300 \mathrm{pc}$ and $200 \mathrm{pc}$. The square represents the apparent scale height derived from the SGP data over a distance range $250 \mathrm{pc} \leq d_{\mathrm{p}} \leq 700 \mathrm{pc}$ plotted at the mean colour of the data.

a: $[1.0,1.4]$, b: $[1.7,2.0]$, c: $[2.13,2.45], \mathrm{d}:[2.45,2.7]$, e: $[2.7,3.07], \mathrm{f}:[3.07,3.45]$, $\mathrm{g}:[3.45,3.82]$, and $\mathrm{h}:[4.0,4.5]$. To each distance distribution we fit an exponential density law and thus deduce the apparent scale height $h_{\text {app }}$. Beyond $d_{\mathrm{p}} \approx 200 \mathrm{pc}$ incompleteness sets in because Malmquist bias leads to an underestimation of distances in apparent magnitude limited samples.

\section{RESULTS}

In Figure 1 we plot $h_{\text {app }}(V-I)$ for different values of $f$. The largest effect is to be seen at the reddest colours with largest $f$. If $f=1$ then at $V-I=4.2, h_{\text {app }}$ reaches a minimum value of $153 \mathrm{pc}$. The detailed behaviour of $h_{\text {app }}(V-I, f)$ is the result of the interplay of the various contributions to the dispersion about the colour-magnitude relation and their variation with colour, as well as the shape of the luminosity function. The effect is largest near the hydrogen burning mass limit because binary systems composed of two main-sequence stars have a mass-ratio very close to unity. 


\section{COMPARISON WITH OBSERVATIONS}

Hawkins (1988) finds that $h_{\text {app }}$ decreases to about $90 \mathrm{pc}$ for his reddest stars with $M_{\mathrm{V}} \approx 14.4$, as shown in Figure 1 . Our model lies in fair agreement with the measurements at $V-I \lessgtr 2.8$ but does not agree with Hawkins' data at redder colours.

Hawkins' measurements indicate a steeper dependence on absolute magnitude which he attributes to a very young and faint population. Stars with colour in the range $3 \leq V-I \leq 3.5$ have $13 \varsigma M \mathrm{~V} \leqslant 14.7$ and, if as young as required by Hawkins, ought to show up as such in other surveys. Giampapa \& Liebert (1987) find no difference in the kinematics of stars fainter than $M_{\mathrm{V}} \approx 12$ as compared to brighter stars, at least down to $M_{\mathrm{V}} \approx 17$. Within $5.2 \mathrm{pc}$, stars in that magnitude range also show space motions representative of an old disc population (Kroupa 1992).

The apparent steepening of the density distribution away from a single exponential is the result of a decreasing maximum sampling distance with decreasing brightness of the stars. Using south galactic pole (SGP) star-count data with $0.93<V-I<2.0$ (Kuijken \& Gilmore 1989b) we measure $h_{\text {app }}$ as a function of sampling distance. In Figure 1 we demonstrate that by applying the same distance completeness limits as Hawkins we find the same decrease of $h_{\text {app }}$, irrespective of colour. These results signify that a single exponential density distribution is not an adequate approximation close to the midplane.

\section{CONCLUSIONS}

Measurements of the apparent galactic disc scale height can be used to study the properties of binary systems if the distance completeness limit is kept constant for all $M_{\mathrm{V}}$.

\section{REFERENCES}

Giampapa, M.S. \& Liebert, J. 1986, $A p J, 305,784$

Gilmore, G. \& Reid, N. 1983, MNRAS, 202, 1025

Hawkins, M.R.S. 1988, MNRAS, 234, 533

Kroupa, P. 1992, Ph.D. thesis, Cambridge University

Kuijken, K. \& Gilmore, G. 1989a, MNRAS, 239, 651

Kuijken, K. \& Gilmore, G. 1989b, MNRAS, 239, 605 